\title{
Acknowledgement to Reviewers of Recycling in 2018
}

\author{
Recycling Editorial Office
}

MDPI, St. Alban-Anlage 66, 4052 Basel, Switzerland

Published: 9 January 2019

Rigorous peer-review is the corner-stone of high-quality academic publishing. The editorial team greatly appreciates the reviewers who contributed their knowledge and expertise to the journal's editorial process over the past 12 months. In 2018, a total of 56 papers were published in the journal, with a median time to first decision of 16 days and a median time to publication of 46.5 days. The editors would like to express their sincere gratitude to the following reviewers for their cooperation and dedication in 2018:

\author{
Achinivu, Ezinne \\ Astiaso Garcia, Davide \\ Balde, Cornelis Peter \\ Bartolacci, Francesca \\ Bassin, Joao Paulo \\ Bates, Margaret \\ Beauson, Justine \\ Behnood, Ali \\ Belbase, Shashidhar \\ Bizyukov, Pavel \\ Boratyński, Filip \\ Brambilla, Massimo \\ Braymand, Sandrine \\ Bufoni, André \\ Buil, Pilar \\ Bujanovic, Biljana \\ Bullock, William \\ Calabrò, Paolo S. \\ Carlos Alberola, Mar \\ Carnero Moya, María Del Carmen \\ Casella, Patrizia \\ Cespi, Daniele \\ Chalioris, Constantin \\ Chen, Guobao \\ Coca, Mónica \\ Colangelo, Francesco \\ Colantoni, Andrea \\ Cole, Christine \\ Coman, Mihaela Denisa \\ Cristiani, Cinzia \\ Cruz, Rebeca \\ Cucchiella, Federica \\ Curtis, Chadwick C. \\ Deng, Yihuan \\ Dhindsa, Kulwinder Singh \\ DiLandro, Luca
}

\author{
Dimcheva, Vanya \\ Dinis, Maria Alzira Pimenta \\ Donatello, Shane \\ Du Plessis, Roelien \\ Elduque, Daniel \\ Engel, David \\ Erdem, Emre \\ Ferronato, Navarro \\ Fewkes, Alan \\ Figueira, Rita \\ Fiore, Silvia \\ Formela, Krzysztof \\ Fujita, Toyohisa \\ Gasparrini, Massimiliano \\ Gómez-Soberón, José Manuel \\ Grbes, Anamarija \\ Grossi, Giancarlo \\ Guo, Zhao-Xia \\ Hanifzadeh, Mohammadmatin \\ Harja, Maria \\ Hettiarachchi, Hiroshan \\ Hsiang, Hsing-I \\ Hung, Yung-Tse \\ Iacovidou, Eleni \\ Ifelebuegu, Augustine \\ Jeong, Bongju \\ Jezierski, Jan \\ Jin, Kailong \\ Jo, Young-min \\ Juszczak, Lesław \\ Kalla, Smail \\ Kelly, Geoff \\ Khan, Amir \\ Kharlov, A.V. \\ Komilis, Dimitrios \\ Konstantakopoulou, Foteini
}


Koumoulos, Elias P.

Kowalczewski, Przemysław

Kowalska, Ewa

Kristjánsdóttir, Helga

Kumar, Amit

Kusch-Brandt, Sigrid

Kuznetsov, Vladimir E.

La Mantia, Francesco Paolo

Ladomersky, Juraj

Lalas, Stavros

Lazaro Nebreda, Jaime

Li, Kai Yuan

Li, Xiaofang

Li, Xiaojun

Liu, Tianyu

Liu, Yanbiao

Lopez, Felix A.

Low, Jonathan Sze Choong

Lyons, Kevin

Mandelli, Stefano

Martinez-Rojas, Enriqueta

Mautner, Andreas

Mavragani, Amaryllis

Messina, Francesco

Mizera, Ales

Morrissey, Anne

Muro Jr, Aldo

Navarro, Francisco Javier

Nuutila, Jaakko

Ojeda-Benitez, Sara

Orac, Dusan

Ozbakkaloglu, Togay

Paladino, Ombretta

Peterson, Karl

Pineda, Paloma

Plush, Sally

Pohar, Andrej

Pomi, Raffaella

Psomopoulos, Constantinos S.

Rada, Elena

Rada, Elena Cristina

Raptopoulos, Grigorios

Regueiro, Araceli

Ribeiro, Maria C.S.
Rodríguez Sáez, Elena

Rogula-Kozlowska, Wioletta

Roy, Sagar

Sakagami, Kimihiro

Sakulich, Aaron

Salinas-Rodríguez, Eleazar

Sarasini, Fabrizio

Scheinberg, Anne

Schneider, Felicitas

Schneider, Petra

Secondi, Luca

Semblante, Galilee

Simoes, Pedro

Smith, York

Song, Kenan

Sundqvist, Jan-Olov

Szymanska-Chargot, M.

Szymańska-Pulikowska, Agata

Taddeo, Raffaella

Teixeira, Paulo

Thomas, Terry

Tonjes, David

Tsagarakis, Konstantinos

Tsantopoulos, Georgios

Tungal, Richa

Udofia, Emilia Asuquo

Valentukeviciene, Marina

Van Loenen, Evert J.

Vogtländer, Joost

Wang, Bairong

Wang, Lu

Wilts, Henning

Yao, Hui

Yeih, Wei-Chung

Yilmaz, Erol

Yonk, Ryan

Yu, Jiayi

ZanniKos, F.

Zarrelli, Armando

Zhang, Hao

Zhang, Wei

Zurbrügg, Christian 